\title{
„Eine Menge von Landschaftsskizzen.“ Zur Bedeutung des Zeichnerischen in Ludwig Wittgensteins Spätphilosophie
}

\author{
Hana Gründler, Florenz
}

„Die philosophischen Bemerkungen dieses Buches sind gleichsam eine Menge von Landschaftsskizzen, die auf [...] langen und verwickelten Fahrten entstanden sind", so beschreibt Ludwig Wittgenstein das Resultat seiner langjährigen Denkbewegungen im Vorwort zu den Philosophischen Untersuchungen. ${ }^{1}$ In den zahlreichen Entwürfen zum Vorwort variierte Wittgenstein das Motiv der ,Gedankenlandschaft ${ }^{`}$ und der Landschaftsskizze etliche Male, wobei jedoch der Grundtenor bestehen blieb. Die Untersuchungen sind eine „Sammlung“ oder „Zusammenstellung“" von Skizzen, eine „Menge“ von „Ansichten“, „Landschaftsskizzen“, die „gezeichnet“ und „entworfen“ werden müssen. ${ }^{2}$ Bezeichnenderweise finden sich nicht nur im Vorwort zu den Untersuchungen, sondern auch in anderen nach 1930 entstandenen Schriften viele Passagen, in denen Wittgenstein Parallelen zwischen seinem philosophischen Denken und künstlerischen Denk- und Verfahrensweisen zieht und sich selbst als einen Maler, allerdings einen recht schlechten, bezeichnet. ${ }^{3}$ Die Tatsache, dass Wittgensteins Beschreibung seiner eigenen, sechzehn Jahre währenden Arbeit, der in dieser Zeitspanne entwickelten Methode und seines Denkens durch die Verwendung einer an der Bildenden Kunst orientierten Sprache gekennzeichnet ist, wurde in der Forschung nur am Rande berücksichtigt. ${ }^{4}$

Dieses mangelnde Interesse mag dem Umstand geschuldet sein, dass die philosophische Relevanz der Metapher gerade im Gegensatz zum begrifflich klaren und eindeutigen Sprachgebrauch immer noch als relativ gering eingestuft wird. Für Wittgenstein selbst spielte die Bildlichkeit der Sprache jedoch eine unübersehbare Rolle. Metaphern wurden von ihm nicht als ein 
die sprachliche Reinheit verunstaltendes Element betrachtet. Vielmehr diente ihm die bildhafte Sprache dazu, festgefahrene Denkmuster zu durchbrechen und den Leser zu neuen Sicht- und Denkweisen anzuregen. ${ }^{5}$ Konsequenterweise muss angenommen werden, dass Wittgenstein die im Vorwort formulierten Bezüge zur Welt der Kunst kaum als zu vernachlässigende Elemente seines Denkens verstanden haben wird. ${ }^{6}$

Aus welchem Grund vergleicht ein Philosoph sowohl die Methode das Skizzieren, das Malen - als auch die Resultate seines Denkens - eine „Menge von Landschaftsskizzen“ oder ein „Album“ - mit denjenigen der Bildenden Kunst? Wieso nennt er den Leser dezidiert einen „Betrachter“ und fordert ihn dazu auf, seine philosophischen Bemerkungen wie ein Album zu durchblättern, in dem Skizze auf Skizze derselben Gedankenlandschaft entworfen wird? Was für Vorstellungen verknüpft Wittgenstein mit der Denkfigur der Zeichnung? Geht es ihm um scharfe Umrisszeichnungen oder um nicht-fixierende Skizzen, und was für inhaltliche Konsequenzen folgen aus dieser Unterscheidung?

Im Folgenden soll diesen Fragen nachgegangen werden, um so der Bedeutung des Zeichnerischen für Wittgensteins Spätphilosophie auf die Spur zu kommen, was nicht in abschließenden Linien, sondern lediglich in tastenden Versuchen geschehen kann. Es scheint sinnvoll, näher zu untersuchen wie, wann und weshalb Wittgenstein Begriffe wie „Strich“, „Kontur“, „Unschärfe“, „Zeichnung“ „Landschaftsskizze“ oder „Album“ einsetzt. Dabei gilt es am Rande auch seine Überlegungen zur Grenze sowie die Verwendung architektonischer Metaphern zu berücksichtigen, die insbesondere im Tractatus logico-philosophicus relevant sind.

Wie gezeigt werden soll, war Wittgenstein in seinen Äußerungen nicht darum bemüht, künstlerische Verfahrensweisen abstrakt-theoretisch zu erläutern, sondern er war diesen Verfahrensweisen affin, hat versucht, sie fruchtbar zu machen und anzuwenden. Die zeichnerische Terminologie scheint eine produktive Funktion in seiner Spätphilosophie zu besitzen: Gerade der Bezug auf das Medium der Zeichnung beziehungsweise der Landschaftsskizze ermöglicht es Wittgenstein unter anderem, die Vorstellung eines fixierten Sehens oder Denkens in Frage zu stellen, das in einer in sich geschlossenen und vollendeten Form ausgedrückt werden kann, die ganzheitlich zu erfassen ist. Der Problematisierung der Kontur, dem Starkmachen (ästhetischer) Polyperspektivität und damit einhergehend die dem Rezipienten zugeschriebene Fähigkeit, die teils verflochtenen ,Gedanken- 
skizzen` weiterzuentwickeln, also eine aktive Seh- und Denkarbeit zu leisten, kommt somit eine wichtige Rolle zu.

Um sich diesem Gedankenkomplex widmen zu können, müssen vorab einige wenige, für die Argumentation wichtige Überlegungen zu Wittgensteins Philosophie vorgenommen werden. Philosophie war für Wittgenstein immer schon Sprachkritik. In der Zeit des Tractatus war ihm daran gelegen, eine formalisierte Zeichensprache herauszuarbeiten, die Zweideutigkeiten, derer die Umgangssprache voll ist, gar nicht entstehen lässt. Es ist eine gleichsam entkleidete Sprache, die das logische Gerüst darlegt. ${ }^{7}$ In den frühen 1930erJahren sollte sich Wittgenstein von dieser Vorstellung abwenden, oder besser gesagt diesen Umgang mit Sprache als ein Sprachspiel unter vielen kennzeichnen. ${ }^{8}$ Er wandte sich einem dynamischen Denken zu und begann, eine Gebrauchstheorie der Sprache zu formulieren, in der die im Medium der Sprache inhärente Relativität, historische Bedingtheit und Kontextabhängigkeit berücksichtigt wird. ${ }^{9}$ Schon in der ersten Definition des Sprachspiels im Blauen Buch war Wittgenstein darauf bedacht, sich den konkreten Fällen zu widmen. Kritisch vermerkt er, dass das „Streben nach Allgemeinheit“, dem beinahe alle Philosophen unterliegen (BB, 39), die Untersuchung des konkreten Gebrauchs von Sprache erschwere. Dieses Streben nach Allgemeinheit ist für Wittgenstein schuld an einer Vielzahl philosophischer Verwirrungen, die nur behoben werden können, wenn ,der Philosoph endlich schaut“ (BB, 39). ${ }^{10}$ Drei Punkte sind von besonderer Bedeutung: 1) Wittgensteins Kritik an der Tendenz zur Verallgemeinerung, 2) seine Betonung des Schauens und 3) seine Bevorzugung eines exemplarisch-analogischen Denken, das den in der Ästhetik und Kunst vorhandenen Denk- und Verfahrensformen sehr nahe ist.

Gemäß Jacques Bouveresse und Judith Genova kann das Sehen zum Paradigma des gesamten Wittgensteinschen Denkens erhoben werden. ${ }^{11}$ Wittgenstein sei der Vorstellung treu geblieben, die philosophische Untersuchung müsse die Sicht auf die Dinge verändern, die Einnahme einer anderen Perspektive ermöglichen. Dies wird durch seine stetigen Hinweise deutlich, dass die meisten Philosophen gar nicht richtig sehen können:

Ich schaute steif vor mich hin - aber nicht auf irgend einen bestimmten Punkt oder Gegenstand. Meine Augen waren weit offen, meine Brauen nicht zusammengezogen (wie sie es meistens sind, wenn ein bestimmtes Objekt mich interessiert). Kein solches Interesse war dem Schauen vorangegangen. Mein Blick war > vacant $<[\ldots] .($ PU 412) 
Der Philosoph wird zum vor sich Hinstarrenden, der die Gegenstände aus den Augen verliert und bestimmte Sehweisen passiv appliziert. ${ }^{12}$ „Die Unruhe in der Philosophie, könnte man sagen, kommt daher, dass wir die Philosophie falsch ansehen, falsch sehen $[\ldots]^{{ }^{“} .{ }^{13}}$ Es ist nun das genaue und aktive Hinsehen, das den Philosophen davor bewahren soll, sich in theoretisch-abstrakte Konstruktionen zu verirren und darüber die „interessante“ Einzelheit zu vergessen, die häufig eine ganze Theorie in Frage stellen kann: „Sage was du willst, solange es dich nicht verhindert, zu sehen, wie es sich verhält. (Und wenn du siehst, wirst du manches nicht sagen.) “14 (PU 79)

In diesem Zusammenhang ist der Begriff der Familienähnlichkeit relevant. Eine erste Vorwegnahme der Idee der Familienähnlichkeit findet sich in dem 1929 gehaltenen Vortrag über Ethik. ${ }^{15}$ Wittgenstein verweist hierbei bezeichnenderweise auf die Galtonsche Komposit-Fotografie: Geht es Galton jedoch um das Herausarbeiten eines Idealtypus, indem er von einer Vielzahl von Gesichtern zu einem gelangt, kehrt Wittgenstein die Sache in Bezug auf die Frage, was die Bedeutung von ,gut" sei, um. Ausgehend von Moores idealer Definition, zeigt er eine Vielzahl von Möglichkeiten auf, den Begriff „gut“ zu verwenden, wobei die Übergänge fließend sind und es keine Identität, sondern lediglich Familienähnlichkeiten zwischen den einzelnen Elementen gibt. Das Konzept der Familienähnlichkeit demonstriert, dass die Mannigfaltigkeit zum Beispiel der Spiele nicht dazu führen darf, ihre Differenzen glätten zu wollen und sie unter dem alles neutralisierenden Begriff erstarren zu lassen. ${ }^{16}$

Zugleich geht damit die Einsicht einher, dass sich die Begriffe nicht mehr exakt eingrenzen lassen. Vielmehr sind sie zu den Rändern hin durchlässig und „verschwommen“. ${ }^{17} \mathrm{Da}$ die Grenzen der Sprache keinen einheitlichen Verlauf haben, können, so Wittgenstein, nur die vielfältigen und situationsspezifischen Verwendungen von Ausdrücken in unserer Alltagssprache beschrieben werden. Dem entgegen übersehen Theorien und Systeme prinzipiell die reale Mannigfaltigkeit der Sprache und der darin ausgedrückten Phänomene. ${ }^{18}$ Dass die alltägliche Sprache nicht von festen, ultimativen Regeln begrenzt ist, bedeutet, dass sie sich in einem permanenten Prozess der Veränderung befindet. Wittgenstein hat diese Dynamik der Worte wie folgt formuliert:

Kannst du die Grenzen angeben? Nein, du kannst welche ziehen: denn es sind noch keine gezogen. [...] »Aber dann ist ja die Anwendung des Wortes nicht geregelt $[\ldots] . \lll-$ Es ist nicht überall von Regeln begrenzt [Hervorhebung Wittgenstein]. (PU 68) 
Die klaren Striche, der Versuch, scharfe Grenzen in der Sprache zu ziehen, erweisen sich als trügerisch. In Paragraph 88 der Philosophischen Untersuchungen stellt sich Wittgenstein die Frage, inwiefern eine abstrakte Erklärung zu verstehen sei als „das Abgrenzen eines Bezirks durch einen Kreidestrich?“ Um zu antworten: „Da fällt uns gleich ein, dass der Strich eine Breite hat.“ (PU 88) Diesen Verweis auf die Breite des Strichs nutzt Wittgenstein, um über die Frage der Unschärfe und der Exaktheit nachzudenken, die er mit der Fotografie in Verbindung setzt, die aber insgesamt mit der Frage nach der Reinheit beziehungsweise der Mehrdeutigkeit und Vielschichtigkeit der Linie verbunden ist. ${ }^{19}$ Die Grenze scheint durch die Breite des Striches zu verschwimmen - deswegen auch die Anspielung auf die „Farbgrenze“-, sie ist nicht mehr ganz exakt zu bestimmen, aber Unschärfen sind für Wittgenstein ebenso wie in der Fotografie je nach Kontext durchaus akzeptabel. Die Offenheit und Veränderbarkeit der Grenzen soll nun aber nicht zur Annahme verführen, die Begriffe seien deswegen nicht zu gebrauchen und ungültig. Wittgenstein hat zu Recht darauf verwiesen, dass die verschwommenen Ränder im Regelfall im alltäglichen Sprachgebrauch gar nicht störend sind. Die exakte, vom Begriffsidealismus angestrebte Grenzziehung wird hingegen als eine Form von Projektion der Kristallreinheit in die alltägliche Sprachpraxis entlarvt. Spiel ist ein Begriff „mit verschwommenen Rändern“, der ähnlich einer unscharfen Photographie „gerade das ist, was wir brauchen“ (PU 71). Es ist diese „Vagheit in den Regeln“ (PU 100), vor der die ,Sprachidealisten', die ihr Ideal in der Wirklichkeit suchen, zurückschrecken. In einem Manuskript von 1947 spricht Wittgenstein bezeichnenderweise davon, dass die Kontur überhaupt nicht dafür geeignet sei, subtile Übergänge und Ähnlichkeiten darzustellen. Sie entspräche eher einem Genauigkeitsideal, das die Gegenstände durch die Kontur zu etwas klar Definiertem und somit Begreifbaren werden lässt: „Wenn man immer alles durch Konturen wiedergibt, kann man einen allmählichen Übergang nicht darstellen. “ (MS 134, S. 2, 1. 3. 1947) ${ }^{20}$

Diese Akzentuierung der Unschärfe und die Problematisierung der Kontur führt uns zur Bedeutung des Zeichnerischen für Wittgensteins Spätphilosophie zurück. Die Nuanciertheit, mit der Wittgenstein die Begriffe einsetzt, spricht für eine Sensibilität gegenüber den vielschichtigen und komplexen Implikationen des Zeichnerischen und der mit ihm einhergehenden Denkweisen. Ein Beispiel: In den vielzähligen Varianten zum Vorwort der Untersuchungen kann nachvollzogen werden, wie sich Wittgenstein graduell von der Idee verabschiedet, seine Bemerkungen seien „eine Menge von Bildern“. ${ }^{21}$ Die Bilder werden zu Skizzen. 
Diese subtilen Verschiebungen werden in den wenigen philosophischen Studien, die sich mit Wittgenstein und der Zeichnung auseinandergesetzt haben, erwähnt, aber nicht eingehender untersucht. ${ }^{22}$ In der Bildwissenschaft hingegen gibt es eine hochgradige Differenzierung des Zeichnungsund des Linienbegriffs und gerade die Thematik der Übergänge und der Offenheit wird in aktuellen Theorien der Zeichnung intensiv diskutiert, was durchaus auch für eine Analyse von Wittgensteins Denken fruchtbar gemacht werden kann. ${ }^{23}$

Die Zeichnung wurde schon seit der Antike bevorzugt mit der Umrisslinie in Verbindung gebracht. Es ist die Kontur, die die Wesenheit der Dinge herauskristallisiert und den geistigen Entwurf des Künstlers sichtbar werden lässt - dies zumindest die Annahme der klassisch-idealistischen disegnoTheorien im 16. Jahrhundert. ${ }^{24}$ Neben dieser Idee der Zeichnung, entwickelte sich Ende des 15. Jahrhunderts eine, die die Kontur in Frage stellte und die nicht-fixierenden Strichbündel ins Zentrum des graphischen Prozesses setzt. Leonardo kommt hierbei eine Vorreiter-Rolle zu, aber die Entwicklung kann über Tizian, Gainsborough, Cézanne bis hin zu Giacometti nachgezeichnet werden (Abb. 1 und Abb. 2). ${ }^{25}$ In Leonardos berühmten componimenti inculti etwa werden verschiedene Entwurfsideen verdichtet, die Linien verwirren sich, es kommt zu Überlagerungen. Dadurch entstehen polysemantische Verflechtungen, die weitergedacht werden können. Seine rasch ausgeführten Skizzen haben keine formelle Grenze, definitive Kontur, sondern sind durch einen formsuchenden Charakter geprägt. Innerhalb der Grenzen des Blattes - hier der Skizze für eine Madonna mit Kind - entspinnt sich ein freier und dynamischer Prozeß, in dem die pentimenti eine Hilfe in Leonardos Suche nach neuen gestalterischen Lösungen sind (Abb. 3). David Rosand betont zu Recht, dass auch Leonardos Verwendung des sfumato in der Malerei in Zusammenhang mit der Infragestellung der Kontur interpretiert werden muss. Das sfumato als Verwischung der Umrisslinie und Verschmelzung von Form und Umgebungsschatten entspricht gemäß Leonardo dem Fehlen graphischer Grenzen in der Natur selbst. ${ }^{26}$ Durch die formale Offenheit der nicht-fixierenden Zeichnung mit ihren verwobenen Strichbündeln wird der Betrachter dazu angeregt, die Darstellung vermittels seines Vorstellungsvermögens aktiv weiterzudenken. Die konturlose Zeichnung erfordert eine kontinuierliche, nie ganz endende Seharbeit, die zugleich eine Denkarbeit ist. Dies hat zum Beispiel Merleau-Ponty eindrücklich in seinen Studien zu Cézanne gezeigt. Cézanne bedient sich selten einer einzigen, strengen Konturlinie, um einen Gegenstand 
Abb. 1

Paul Cézanne

Studie eines weiblichen Aktes

(1877-1879)

Bleistift auf Papier, $21 \times 18,7 \mathrm{~cm}$

Basel, Öffentliche Kunstsammlung, Kupferstichkabinett

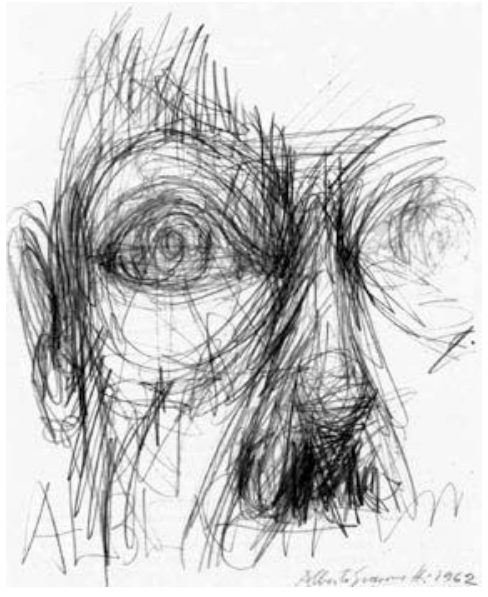

Abb. 2

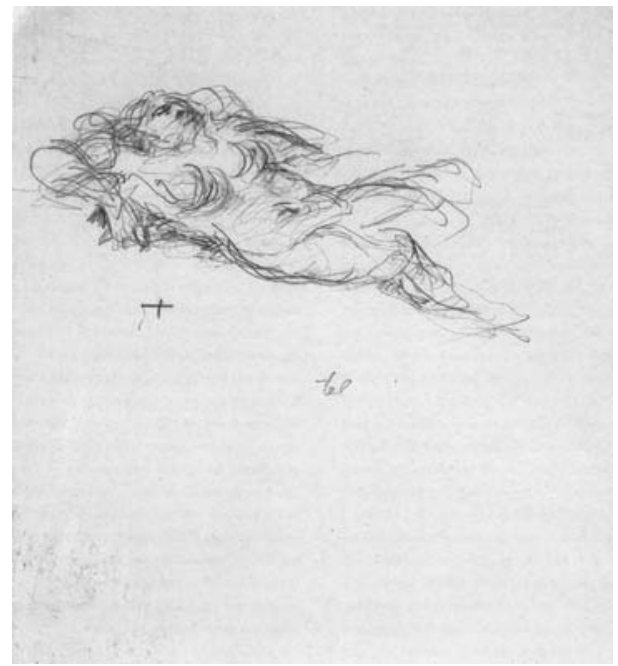

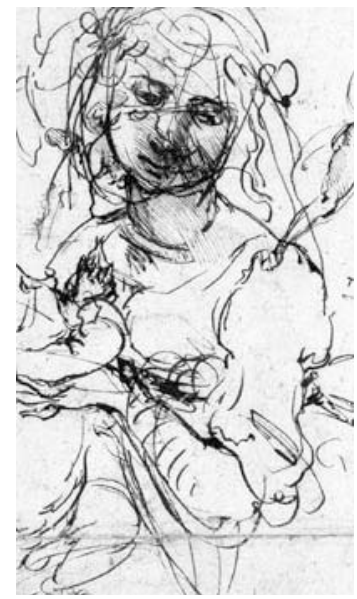

Abb. 3

Leonardo da Vinci Studie für eine Madonna mit Kind und Heiligem Johannes (Detail) (ca. 1478)

Tusche auf Papier, 40,5 × $29 \mathrm{~cm}$ Windsor, Royal Library, RL 12276r 
darzustellen, sondern mehrfacher Konturen. Es ist ein contour naissant, eine Kontur im Akt des Werdens, nicht eine bereits vollendete. ${ }^{27}$ Seine Zeichnungen, die häufig einen unvollendeten Charakter aufweisen, präsentieren demzufolge nicht mehr eine feststehende Bildordnung, die das Auge des Betrachters beinahe mühelos rezipieren kann, vielmehr gewinnt die Zeichnung an sich einen Erkundungswert (Abb. 1). Wie gleich noch näher gezeigt wird, verwendet Wittgenstein die Denkfigur der Zeichnung bezeichnenderweise, um auf den unabschließbaren Charakter seines Denkens/Werkes zu verweisen und gleichzeitig die Wichtigkeit des aufmerksamen Betrachtens zu akzentuieren. Sie scheint für den Wittgenstein der Untersuchungen weniger aufgrund ihrer Assoziation mit der Kontur als vielmehr aufgrund der Möglichkeit der unendlich flexiblen, offenhaltenden Beschreibung, der Überlagerungen und Verdichtungen so wichtig zu sein. Seine Vorstellung von Zeichnung korreliert mit der rohen Skizze, dem unvollendeten Entwurf, der nicht das Allgemeine, sondern das Spezifische eines ästhetischen Objekts einzufangen versucht und in seiner ,Beschreibung' besonders flexibel ist. Damit geht auch einher, dass es nicht die eine, richtige Sicht auf den Gegenstand gibt, sondern multiple und variable Perspektiven. Philosophische und künstlerische Werke, die durch diesen offenen Charakter geprägt sind, erfordern vom Rezipienten eine intensive und kreative Arbeit des Sehens und Denkens. In jüngeren Studien zur Zeichnung und zur Linie werden diese Aspekte besonders stark gemacht: Nach Norman Bryson und Gottfried Boehm ermöglicht es die Offenheit der Zeichnung den verschiedenen graphischen Spuren nachzugehen, was beim Betrachter stärker als bei der geschlossenen Oberfläche des Gemäldes das Gefühl hervorruft, den Akt des Zeichnens nachzuvollziehen. ${ }^{28}$ Gerade mit der Skizze gehen epistemische und kreative Möglichkeiten einher, die das Prozessuale in der Weltaneignung hervorheben. Hubert Damisch sensibilisiert in seinem Traité du trait für diese Offenheit und Polysemie der Zeichnung. Er zeigt, dass der Ursprung der westlichen Zeichnung eigentlich das Nachtasten eines Schattens sei. Keine scharf abgrenzende Linie, sondern vielmehr ein wiederholt ansetzender Strich ist hier am Werk. In der westlichen Tradition der disegno-Theorie mit ihrem Ideal einer klaren, souverän gezogenen Konturlinie, so Damisch, werden die Vielzahl der Striche und ihre intrinsische Mannigfaltigkeit nun aber vereinheitlicht und idealisiert. ${ }^{29}$

Ausradierung der Entstehung und des Werdens? Idealisierungen der Linie und Idealisierungen der Sprache? Träume nach der scharfen Kristallreinheit des Zeichnens und Denkens? Wie bereits erörtert, wird die exakte, vom Begriffsidealismus angestrebte Grenzziehung von Wittgenstein als eine Form 
von Projektion der Kristallreinheit in die alltägliche Sprachpraxis entlarvt. Dem Ideal einer exakten Grenzziehung in der Sprache setzt er ein „,kompliziertes Netz von Ähnlichkeiten, die einander übergreifen und kreuzen“ (PU 66), vielfältige und verschwommene Striche gegenüber, die er auch in seinem sprachlichen Duktus evoziert. Die Kontur und die Grenze werden von ihm nicht mehr idealisiert, sondern problematisiert. ${ }^{30}$ Der Begriff der Familienähnlichkeit tritt in den Untersuchungen anstelle desjenigen der Grenze im Tractatus.

Im Tractatus ist der häufige Gebrauch konstruktionstechnischer bzw. architektonischer Metaphern auffällig. ${ }^{31}$ Die Grenze und die Vorstellung von klaren Strichen, die diffuse Felder unterteilen, spielen hierbei eine zentrale Rolle: „Die Philosophie soll die Gedanken, die sonst, gleichsam, trübe und verschwommen sind, klar machen und scharf abgrenzen." (TLP, 4.112) Wie es Matthias Kroß treffend formuliert hat, verlangt „das Selbstverständnis des Tractatus [...] nach einem in jedem Detail sicheren Strich. Ein Strich der abgrenzt, der in einem halbwegs bekannten Gebiet gezogen wird, das ein diffuses Feld ist und der Klärung nach innen hin bedarf.“"32 Diese Möglichkeit einer klaren, scharfen ,Linienziehung' und räumlichen Aufteilung erinnert an das Vorgehen eines Ingenieurs oder Architekten bei der Gestaltung einer Blaupause und ist stets auch mit einer spezifischen Form von Perspektive verbunden. Hans Sluga schrieb über den Tractatus, dass , [...] sich die Struktur der Welt so von der Form der Sprache wie von einer Blaupause ablesen“ lasse. ${ }^{33}$ Die Blaupause ermöglicht den geklärten, sozusagen körperlosen Blick von außen auf die Geometrie eines Hauses - das Haus bleibt flach, eine Zeichnung der Kontur, des Gegenübers. Bezeichnenderweise lassen sich in Wittgensteins nach 1929, also nach dem Bau des Palais StonboroughWittgenstein entstandenen Schriften viele Passagen finden, in denen er sich von einer Vorstellung des Sehens und der Perspektive entfernt, wie er sie noch im Tractatus vertrat.

Das Konzept der Perspektive war für sein gesamtes Schaffen zentral. Allerdings verändert sich seine Auffassung in der Früh- und der Spätphilosophie grundlegend. Im Tractatus ging er unter dem Einfluss Spinozas und insbesondere Schopenhauers noch davon aus, dass es die eine richtige Perspektive gäbe, die Betrachtungsweise sub specie aeternitatis, die uns durch die ästhetische Kontemplation, aber auch durch das philosophische Denken ermöglicht wird. ${ }^{34}$ Ab 1930 relativiert er diese Position. Er gibt die richtige Perspektive zugunsten der Polyperspektivität auf, und die ästhetische Betrachtungsweise besteht nun nicht mehr in der Fähigkeit der Dekontextualisierung 
und Kontemplation, sondern darin, Gegenstände immer wieder neu, unter anderen Perspektiven zu betrachten, ein Punkt, der noch näher analysiert wird. Anders als im Tractatus geht es Wittgenstein in seinen späteren Schriften weniger um den Versuch von klaren Grenzziehungen, um die Rekonstruktion der Welt in ihrer Gesamtheit, angefangen bei den einfachen Namen, den Grundbausteinen des Sprachbaus, als darum, verschwommenen Rändern, verwinkelten Gassen, „zick-zack“ Anordnungen und vernetzten Übergängen in der Sprache nachzuspüren. Diese können unmöglich auf einen Blick erfasst werden. ${ }^{35}$ Der Anspruch auf eine unmittelbare und schematische Erfassung der Mannigfaltigkeit der lebendigen und dynamischen Alltagssprache wird von Wittgenstein als Illusion entlarvt, denn der lineare und „direkte Weg ist uns immer versperrt.“ „Die Sprache ist ein Labyrinth von Wegen. Du kommst von einer Seite und kennst dich aus; du kommst von einer andern zur selben Stelle, und kennst dich nicht mehr aus." (PU 203) Statt auf die Welt bzw. die Sprache zu schauen, wollte er nun, wie er selbst schrieb, in die Welt hinabsteigen, ,zurück auf den rauhen Boden“ (PU 107) und sich den Gegenständen seiner Untersuchungen aus verschiedenen Perspektiven nähern.

Das Vorwort der Philosophischen Untersuchungen, in dem Wittgenstein auch auf seine ,zeichnerische“ Methode hinweist, ist paradigmatisch für diesen neuen Ansatz. Indem Wittgenstein seine philosophischen Bemerkungen als eine Menge von Landschaftsskizzen charakterisiert, evoziert er eine optische Wanderstruktur: Er scheint nicht wie ein Kartograph vorzugehen, der die Welt gleichsam aus dem Vogelflug betrachtet und abstrahierend symbolisch zu erfassen versucht. Vielmehr nennt er sich einen Zeichner, der sich in die Gedankenlandschaft begibt, Perspektiven auffächert und immer verschiedene Skizzen von derselben Landschaft, allerdings immer wieder von unterschiedlichen Punkten her entwirft. ${ }^{36}$

Die ursprüngliche Idee, ein zusammenhängendes Buch zu schreiben, in dem Überlegungen zu Fragen der Bedeutung, des Verstehens, des Satzes und vielem anderem zu einem ,lückenlosen Ganzen“ zusammengeschweißt worden wären, hatte Wittgenstein im Laufe der Jahre aufgegeben. Wie Alois Pichler gezeigt hat, wurde sein Unbehagen bezüglich einer Systematisierung der eigenen Gedanken ab 1936 immer offensichtlicher. Seine ursprüngliche, lineare Vorgehensweise empfand er als zu steif: „The form of presentation I started with is not elastic enough. The connections of the exposition are too stiff \& do not leave room for many others which are also present", so Wittgenstein in einer Notiz von 1937. Wurde die Schwierigkeit zur systematischen Anordnung in sehr frühen Schriften noch als persönliche Unfähig- 
keit und Mangel betrachtet, siedelte es Wittgenstein gemäß Pichler später im Gegenstandsbereich selbst an. ${ }^{37}$

,[Die Natur der Untersuchungen] nämlich zwingt uns ein weites Gedankengebiet kreuz und quer, nach allen Richtungen hin zu durchreisen", wobei immer wieder neue Skizzen entworfen werden, die, wie er selbstkritisch vermerkt, alle „mit den Mängeln eines schwachen Zeichners behaftet“ sind. (PU, Vorwort) Der Betrachter, wie Wittgenstein den Leser der Untersuchungen dezidiert nennt, wird sozusagen dazu eingeladen, sich im Bild/Text wandernden Blickes den Details zu nähern und somit die Vielfältigkeit der Welt visuell zu erleben. Er sollte die Landschaft selbst durchwandern, um zu neuen Ansichten zu gelangen, denn er hat nicht den Anspruch auf eine vollständige und einzig korrekte Wiedergabe derselben. Das, was die Leser der Untersuchungen zu sehen bekommen, sind Details, „Ausschnitte einer ungeheuren Landschaft“ (VB 1946, 529), und das Bild, das sich dem „Betrachter“ eröffnet, ist somit niemals ein endgültiges, fixiertes. Und so sind die Untersuchungen, die aus einer Anzahl von „halbwegse[n]“, beschnittenen Bildern bestehen, „eigentlich nur ein Album“ geworden. Durch die auf „verwickelten Fahrten“ entstandenen Skizzen kreiert Wittgenstein die Vorstellung von nicht-fixierenden Linien, die durch ihre Offenheit und ihren umkreisenden, verwobenen Duktus charakterisiert sind. ${ }^{38}$ Sie sind das visuelle Pendant zu seiner eigenen ,offenhaltenden` Denkweise, die auf das Engste mit seiner Schreibweise verbunden ist.

Wirft man einen Blick auf Wittgensteins Werk, wird man schnell feststellen, dass es aus einer unendlichen Anzahl von „Zetteln“, verstreuten Bemerkungen, tagebuchartigen Einträgen und unsystematischen Fragmenten besteht, die selten einen einheitlichen, abgeschlossenen Charakter aufweisen. Vielmehr sind sie untereinander vernetzt und stellen eine Form von ,work in progress ${ }^{6}$ dar. ${ }^{39}$ Es sind ,Gedankenkomplexe', die einen wechselnden, jedoch nie völlig verschiedenen Inhalt aufweisen, da Wittgenstein ständig um dieselben Problemstellungen kreiste. „Ich bewege mich fortwährend im Kreis um das Problem herum. Scheinbar ohne ihm näher zu kommen." (MS 106, S. 30). Wie es Rudolf Haller so schön umschrieben hat, erhält bei Wittgenstein ,nahezu kein Satz und kein Absatz von vornherein einen angestammten Platz [...] jeder ist ein Stück für sich verschieb- und veränderbar, wie unsere lebendigen Gedanken. “40

Wittgensteins skizzierende Vorgehensweise und die „kreuz- und quer“Anordnung seiner Gedanken führt dazu, dass die Behandlung der Themen 
ineinander übergeht, ohne dass die Diskussion der einzelnen Themen jeweils völlig abgeschlossen wäre. Der Leser sieht sich deshalb mit einem komplexen Gedankenteppich konfrontiert, der es erfordert, dass er seine Aufmerksamkeit - ebenso wie bei der Betrachtung eines nicht fixierenden, verwobenen Zeichenduktus - nicht auf einzelne Stellen fixiert, sondern sie schweifen lässt und verschiedene Perspektiven einnimmt.

Dabei ist es für Wittgenstein grundlegend, dass man sich im Denken der Beschränktheit einer Perspektive bewusst wird und sich darum bemüht, die Standpunkte zu wechseln und die verschiedenen daraus resultierenden ,Einsichten` zueinander in Beziehung zu setzen. Das, was dabei zustande kommt, ist nicht etwa das vom objektiven Wissen angestrebte richtige Bild einer Situation, die Summe aller Teile, die zur erwünschten ,Ganzheit führen. Vielmehr erscheint dieses Wissen als ein Kompendium verschiedener Anschauungen und Annäherungen, die zwar ein Ganzes andeuten, sich aber aufgrund der Komplexität sowie der multiplen und variablen Beziehungen immer Modifikationen ausgesetzt sehen.

Wittgensteins ,ästhetischer Perspektivismus' bringt die Frage mit sich, ob es nicht notwendig sei, eine Form von Metaperspektive einzunehmen, von der aus dann die unterschiedlichen Perspektiven, die vielen verstreuten, auf langen Fahrten durch die Landschaft entstandenen Skizzen, zu einem einheitlichen Bild zusammengefügt werden. In der Tat hat Wittgenstein geschrieben, dass der Begriff der übersichtlichen Darstellung von grundlegender Bedeutung für ihn sei. „Er bezeichnet unsere Darstellungsform, die Art, wie wir die Dinge sehen." (PU 122) Wie Alois Pichler und Judith Genova gezeigt haben, muss die übersichtliche Darstellung aber nicht mit der Sicht von oben, der vogelgleichen Perspektive gleichgesetzt werden. Vielmehr plädieren sie dafür, dass die synoptische Sichtweise dazu führe, Zusammenhänge zu sehen, Dinge nebeneinander zu stellen und die Welt von verschiedenen Perspektiven aus zu betrachten. ${ }^{41}$ Beide Autoren gehen jedoch nicht näher darauf ein, dass es gerade die ästhetische Darstellungsform ist, die es ermöglicht, sich für einen Polyperspektivismus einzusetzen, ohne in vollkommener Beliebigkeit zu versinken. ${ }^{42}$

Durch das Offenlegen verschiedener Perspektiven oder gar das Generieren neuer Perspektiven, kann auf die unerschöpfliche Mannigfaltigkeit der Erscheinungen verwiesen werden. Dadurch wird aber auch ersichtlich, dass der Gedanke einer apodiktischen Wahrheit nicht mehr haltbar ist. Wittgenstein betont in seiner mittleren bzw. späten Philosophie, dass Sprache (aber auch Kunst) nicht Wiedergabe von Wesenheit, ${ }^{43}$ sondern vielmehr Interpretation 
ist und Wahrheit aufgrund dessen nicht als metaphysisches Konzept, sondern als durch die Sprachpraxis, die Interpretation und den Kontext Bedingtes und Erlerntes verstanden werden sollte.

Er hat dieses Problem wie folgt beschrieben: „Wie kann man durch Denken die Wahrheit lernen? Wie man ein Gesicht besser sehen lernt, wenn man es zeichnet.“ (Z 255) Gemäß Chris Bezzel sensibilisiert diese Analogie dafür, dass es nicht das Bild eines Gesichts gibt, genauso wenig, wie es die eine Wahrheit gibt. Vielmehr können nur stetig neue Zeichnungen gemacht werden, in denen man sich der Wahrheit lediglich annähern kann. ${ }^{44}$ Wenn man ein Gesicht zeichnet, wird man vertrauter mit seinen Eigenheiten und Details, und gleichzeitig bleibt die Beschreibung besonders flexibel. Bezeichnenderweise dient Wittgenstein der Verweis auf das Medium der Zeichnung in den Vorlesungen über Ästhetik dazu, auf die nicht-subsummierbare Vielfalt der Eindrücke und der damit einhergehenden Interpretationen zu verweisen. Die Fähigkeiten eines Zeichners, ein Gesicht durch vier Striche zu schaffen und dabei eine „unzählbare Anzahl von Ausdrücken zu erzeugen“, sind für Wittgenstein das gelungene Beispiel einer möglichst flexiblen Erfassung einer Situation. ${ }^{45}$ Der Philosoph sollte nun ebenso wie der Zeichner sein Auge an konkreten Problemen üben und schärfen, damit er sich von konventionellen Seh- bzw. Denkweisen sowie von vorgefertigten Konzepten befreien kann und aktiv denkt. „Das Denken ist ganz mit dem Zeichnen von Bildern zu vergleichen", so Wittgenstein. ${ }^{46}$ (PG, S. 163)

In diesem Zusammenhang ist es wichtig, dass Wittgenstein im Vorwort der Untersuchungen nicht nur auf die Zeichnung als vollendetes künstlerisches Produkt eingeht. Vielmehr wird entworfen, gezeichnet, verzeichnet und beschnitten. Dadurch wird gezielt der aktive Prozess des Zeichnens thematisiert und dabei gleichsam die Verbindung von Zeichenakt, Sehakt und Denkakt bestärkt. Die vielfältigen Varianten desselben, die unterschiedlichen Skizzen, verweisen auf die Mobilität des Denkens, die letztlich auch einen infiniten Schreibprozess mit sich bringt. Das von Wittgenstein durchgeführte Beschneiden von Bildern verdeutlicht, wie sehr seine Sicht auf das weite „Gedankengebiet" durch einen persönlichen Zugriff geprägt ist, der nicht verhüllt werden kann und sollte, sondern vielmehr offen gelegt und als grundlegendes Konstituens unserer Sicht und damit einhergehend unsere Erkenntnis von Welt verstanden werden muss. Der Künstler/Philosoph artikuliert, ja, er generiert Perspektiven und beansprucht nicht, dass die von ihm gezeigte Perspektive die einzig richtige ist. Die im Vorwort formulierten Überlegungen 
sind also keineswegs nur eine Kritik oder Legitimation der eigenen Methode. Die unter anderem von Savigny vertretene These, dass der Leser der Untersuchungen die verstreuten Skizzen zu jenem lückenlosen Ganzen zusammenschweißen müsse (vgl. PU, Vorwort), das Wittgenstein zu Beginn seiner Arbeit an den Untersuchungen angestrebt hatte, dabei sozusagen seine Unfähigkeit überbrückend und seine Methode ignorierend, scheint vor dem hier angerissenen Hintergrund nicht sonderlich plausibel. ${ }^{47}$ Die verbale Evokation des Skizzenhaften und die Einladung zum Durchwandern der ,gezeichneten Gedankenlandschaft dient vielmehr zur ,Einstimmung' des Lesers und sensibilisiert dafür, wie man sich den Untersuchungen, die wie „,grobe künstlerische Skizzen sind ${ }^{\text {‘48 }}$, nähern soll. Würde Wittgenstein ein vollendetes Bild evozieren, sozusagen eine geschlossene Bildoberfläche, die eben keinen Einblick in den Denkakt/Zeichenakt mit seinen unendlichen Variationen und vielfältigen, sich überkreuzenden Gedankenbündeln ermöglichen würde, hätte der Leser von vornherein eine falsche Erwartung. Die Landschaftsskizzen und das Album sind Bilder für die Bewegung des Denkens im Schreiben. Der Sehende und der Verstehende werden in Wittgensteins Werk dazu angeregt, sich aktivpoietisch und nicht passiv-rezipierend mit der Welt zu beschäftigen und somit neue Sinnzusammenhänge zu entdecken, denn Ziel seines Philosophierens ist es nicht „Andern das Denken [zu] ersparen. Sondern, wenn es möglich wäre, jemand zu eigenen Gedanken an[zu]regen“. (PU, Vorwort) 


\section{Anmerkungen}

1 Ludwig Wittgenstein, Philosophische Untersuchungen (1984-1989; sechzehnte Auflage, Frankfurt am Main: Suhrkamp Verlag, 2004), Werkausgabe Bd. 1, 225-580, hier 231-233. Im Folgenden zitiert als PU mit Paragraph.

$2 \mathrm{Zu}$ den Entwürfen des Vorwortes siehe Alois Pichler, Wittgensteins Philosophische Untersuchungen. Vom Buch zum Album (Amsterdam/New York: Rodopi Verlag, 2004).

3 „Und ich bin im Grunde genommen doch ein Maler, und oft ein sehr schlechter Maler", so Wittgenstein in einem Fragment von 1949. (Ludwig Wittgenstein, Vermischte Bemerkungen, 1984-1989; sechzehnte Auflage Frankfurt am Main: Suhrkamp Verlag, 2004, Werkausgabe Bd. 8, 445-573, hier 567; im Folgenden zitiert als VB.) Ähnlich im Vorwort, in dem er betont, dass eine Unzahl von ihm geschaffenen Bildern ,verzeichnet, oder uncharakteristisch, mit allen Mängeln eines schwachen Zeichners behaftet" waren (PU, Vorwort, 231). Grundlegende ästhetische Interpretationsansätze sind etwaVirgil Aldrich, „Pictorial Meaning, Picture Thinking, and Wittgenstein's Theory of Aspects, “ Mind 67 (1958): 70-79; Richard Wollheim, Art and its Objects: With Six Supplementary Essays (Cambridge: Cambridge University Press, 1980) erste Auflage 1968; Stanley Cavell, Must We Mean What We Say? (Cambridge/New York: Cambridge University Press, 1969); William G. Lycan, „,Gombrich, Wittgenstein and the Duck-Rabbit, “ Fournal of Aesthetics and Art Criticism 30 (1971): 229-237; Jacques Bouveresse, Wittgenstein. La rime et la raison. Science, éthique et esthétique (Paris: Éditions de Minuit, 1973); Jörg Zimmermann, Wittgensteins sprachphilosophische Hermeneutik (Frankfurt am Main: Klostermann, 1975); Rudolf Haller, „Das Kunstwerk als Gegenstand sub specie aeternitatis,“ in Ästhetik. Akten des 8. Internationalen Wittgenstein-Symposiums, herausgegeben von Rudolf Haller(Wien:Hölder-Pichler-Tempsky, 1984), 30-35; ManfredFrank,,,Wittgensteins Gang in die Dichtung“, in Wittgenstein: Literat und Philosoph, herausgegeben von Manfred Frank und Giancarlo Soldati (Pfullingen: Verlag Günther Neske, 1989), 7-72; Joachim Schulte, Chor und Gesetz. Wittgenstein im Kontext, (Frankfurt am Main: SuhrkampVerlag, 1990); Ben R. Tilghman, Wittgenstein, Ethics and Aesthetics. The View from Eternity (Albany: State University of New York Press, 1991); Felix Gmür, Ästhetik bei Wittgenstein. Über Sagen und Zeigen (Freiburg-München: Verlag Karl Alber, 2000).

4 Die Bedeutung, die dem ästhetisch-kreativen Denken sowie dem visuellen und bildnerischen Vokabular in Wittgensteins Philosophie zukommt, kann nicht zu genüge betont werden. Zu Recht schreibt Peter B. Lewis in Wittgenstein, Aesthetics and Philosophy, dass gerade eine Berücksichtigung der Ästhetik klärend für das 
Verständnis der gesamten Wittgensteinschen Philosophie sein kann. Bezeichnenderweise zieht sich die Kritik an der bisherigen Wittgenstein-Rezeption, der mangelndes Interesse für die ästhetischen Aspekte von dessen Werk vorgeworfen wird, wie ein roter Faden durch die jüngere Forschung. Vgl. etwa Wittgenstein, Theory and the Arts, herausgegeben von Richard Allen und Malcolm Turvey (London/New York: Routledge, 2001); Wittgenstein und die Metapher, herausgegeben von Ulrich Arnswald/Jens Kertscher/Matthias Kroß (Berlin: Parerga, 2004); The Literary Wittgenstein, herausgegeben von John Gibson und Wolfgang Huemer (London /New York: Routledge, 2004); Wittgenstein, Aesthetics and Philosophy, herausgegeben von Peter B. Lewis, (Aldershot: Ashgate, 2004); Wittgensteinkunst, herausgegeben von Fabian Goppelsröder (Zürich/Berlin: Diaphanes, 2006); „Ethik und Ästhetik sind Eins.“ Beiträge zu Wittgensteins Ästhetik und Kunstphilosophie, herausgegeben von Wilhelm Lütterfelds und Stefan Majetschak (Frankfurt am Main: Peter Lang Verlag, 2007); Hana Gründler, Wittgenstein. Anders Sehen. Die Familienähnlichkeit von Kunst, Ästhetik und Philosophie (Berlin: Trafo Verlag, 2008).

5 Zur Bedeutung der bildhaften Sprache in Wittgensteins Denken siehe zum Beispiel Arnswald/Kertscher/Kroß, Metapher (wie in Anm. 4); Frank, Dichtung (wie in Anm. 3); Gibson/Huemer, Literary Wittgenstein (wie in Anm. 4); Pichler, Album (wie in Anm. 2); Zimmermann, Hermeneutik (wie in Anm. 3). Ferner Gmür, Ästhetik bei Wittgenstein (wie in Anm. 3), Kap. 3.3 „Das Metaphorische“.

6 Chris Bezzel, Judith Genova, Alois Pichler und Rüdiger Zill gehören zu den wenigen, die in ihren Studien auf die Bedeutung zeichnerischer und künstlerischer Metaphern verwiesen haben. So schreibt Pichler, der in seinem erhellenden Buch den diffizilen Entstehungsprozess der Philosophischen Untersuchungen sowie Wittgensteins damit einhergehende methodische Veränderung anhand der vielfältigen Manuskripte nachzeichnet, und dabei unter anderem auf die Bedeutung des Fragments und der bildhaften Sprache eingeht, dass die Philosophischen Untersuchungen „interessante, neue Bezugspunkte zu den Welten des Künstlers“ beinhalten würden. Er führt diese Bezugspunkte jedoch nicht näher aus, obgleich er immer wieder zeichnerische Metaphern thematisiert. Chris Bezzel, Wittgenstein zur Einführung (Hamburg: Meiner, 2000); Judith Genova, Wittgenstein. A Way of Seeing (London/New York: Routledge, 1995); Pichler, Album (wie in Anm. 2), 73. Ferner Rüdiger Zill, „Der Vertrakt des Zeichners. Wittgensteins Denken im Kontext der Metapherntheorie“, in Wittgenstein und die Metapher, herausgegeben von Ulrich Arnswald/Jens Kertscher/Matthias Kroß (Berlin: Parerga, 2004), 137-164.

7 Ludwig Wittgenstein, Tractatus logico-philosophicus (1984-1989; sechzehnte 
Auflage Frankfurt am Main: Suhrkamp Verlag, 2004), Werkausgabe Bd. 1, 7-86. Im Folgenden zitiert als TLP mit Paragraph. Vgl. hierzu TLP 4.002. David Stern hat in seinem Aufsatz ,The Methods of the Tractatus. Beyond Positivism and Metaphysics?" die fünf grundlegenden Tractatus-Lesarten der letzten achtzig Jahre herausgearbeitet, so 1) die logisch atomistische Lesart (Russell, Ramsey), 2) die logisch positivistische Lesart (Neurath, Carnap, Schlick), 3) Die metaphysische Lesart (Anscombe, Stenius), 4) die irrationalistische Lesart (Engelmann, Janik und Toulmin) und 5) die therapeutische Lesart (Winch, Diamond etc). Vgl. David Stern, „The Methods of the Tractatus. Beyond Positivism and Metaphysics?", in Logical Empiricism. Historical and Contemporary Perspectives, herausgegeben von Paolo Parrini/Wesley Salomon/Merrilee Salomon (Pittsburgh: University of Pittsburgh Press, 2003), 126-132.

8 Anthony Kenny hat im letzten Kapitel seines 1972 erstmals erschienenen Buches Wittgenstein auf die Einheit von Wittgensteins Philosophie hingewiesen und die bis dahin üblichen Tendenzen, eine beinahe unüberwindbare Kluft zwischen Frühund Spätphilosophie zu konstruieren, scharf kritisiert. Anthony Kenny, Wittgenstein (Frankfurt am Main: Suhrkamp Verlag, 1974), insb. Kapitel 12 „Die Einheit der Philosophie Wittgensteins“. Ferner Zimmermann, Hermeneutik (wie in Anm. 3), 87-88.

9 Vgl. hierzu etwa Kenny, Wittgenstein, 259 und René Heinen, Sprachdynamik und Vernunft. Untersuchungen zum Spätwerk Nietzsches und Wittgensteins (Würzburg: Königshausen \& Neumann, 1998).

10 Ludwig Wittgenstein, Das Blaue Buch (1984-1989; sechzehnte Auflage Frankfurt am Main: Suhrkamp Verlag, 2004), Werkausgabe Bd. 5, 15-116. Im Folgenden zitiert als BB. Der im Blauen Buch angefangene Denkweg, hin zur Pluridimensionalität des Sprachspielbegriffs in den Philosophischen Untersuchungen, akzentuiert das jeweils Einzigartige eines Sprachspiels und versucht eben nicht, erklärende Urteile zu fällen, sondern rein deskriptiv vorzugehen, sich also konkrete Fälle vorzunehmen, um anhand derer, den Gebrauch von Sprache besser nachvollziehen zu können. Vgl. hierzu Heinen, Sprachdynamik (wie in Anm. 9) sowie Heinz Spremberg, Zur Aktualität der Ästhetik Immanuel Kants. Ein Versuch zu Kants ästhetischer Urteilstheorie mit Blick auf Wittgenstein und Sibley (Frankfurt am Main: Peter Lang Verlag, 1999), 190.

11 Dass die Metaphern des Schauens, des Blickes und der Perspektive bereits im Tractatus logico-philosophicus eine grundlegende Rolle besaßen, konnte Bouveresse in seinem 1971 erschienenen Buch Wittgenstein. La rime et la raison. Science, éthique et ésthetique nachweisen. Jacques Bouveresse, Wittgenstein. La rime et la raison. Science, 
éthique et esthétique (Paris: Éditions de Minuit, 1973); Jacques Bouveresse, Essais III. Wittgenstein ou les sortilèges du langage (Marseille: Agone, 2003). Ferner grundlegend Genova, $A$ Way of Seeing (wie in Anm. 6).

12 Vgl. hierzu Rolf Wiggershaus, Wittgenstein und Adorno. Zwei Spielarten modernen Philosophierens (Göttingen: Wallstein Verlag, 2000), 44-47.

13 Ludwig Wittgenstein, Zettel (1984-1989; sechzehnte Auflage Frankfurt am Main: Suhrkamp Verlag, 2004), Werkausgabe Bd. 8, 259-443. Im Folgenden zitiert als Z. Hier Z 447.

14 Vgl. hierzu den Aufsatz von Daniel Eckert, „Wittgenstein, die Ästhetisierung der Philosophie und der Relativismus“, in Ästhetik. Akten des 8. Internationalen WittgensteinSymposiums, herausgegeben von Rudolf Haller (Wien: Hölder-Pichler-Tempsky, 1984), 36-38.

15 Ludwig Wittgenstein, „Vortrag über Ethik“, in Vortrag über Ethik und andere kleine Schriften, herausgegeben von Joachim Schulte (Frankfurt am Main: Suhrkamp Verlag, 1989), 7-19.

16 Wittgensteins Bemerkungen zum Begriff der Familienähnlichkeit scheinen den Leser davon überzeugen zu sollen, dass eine exakte, theoretische Definition dessen, was ein Sprachspiel ist, nicht notwendig ist, da die Diskussion mittels konkreter Beispiele, wie sie in den ersten sechzig Paragraphen der Untersuchungen stattgefunden hat, ausreicht. Vgl. hierzu etwa Richard W. Beardsmore, „Art and Family Resemblances,“ Philosophical Investigations 18 (1995): 199-215. Ferner Hans Sluga, „Family Resemblance,“ Grazer Philosophische Studien 71 (2006): 1-21.

17 Bei Wittgenstein ist ähnlich wie bei Nietzsche eine anti-essentialistische Tendenz zu erkennen, da der Begriffsidealismus durch den Verweis auf konkrete Fälle eingeschränkt wird und die Differenzen der Dinge nicht mehr zugunsten einer idealisierten Übereinstimmung in der Sache geglättet werden dürfen. Vgl. Heinen, Sprachdynamik (wie in Anm. 9), 27.

18 Vgl. Spremberg, Zur Aktualität der Ästhetik Immanuel Kants (wie in Anm. 9), 190.

19 Vgl. hierzu auch den Aufsatz von Rüdiger Zill. Zill, „Vertrakt des Zeichners“ (wie in Anm. 6), 154-155.

20 Vgl. hierzu Pichler, Album (wie in Anm. 2), 205 ff.

$21 \mathrm{Zu}$ den verschiedenen Entwurfsstadien des Vorwortes siehe Pichler, Album (wie in Anm. 2).

22 Siehe Anmerkung 6.

$23 \mathrm{Zu}$ aktuellen Theorien der Zeichnung siehe exemplarisch folgende Publikationen: James Elkins, „Marks, Traces, Traits, Contours, Orli, and Splendores”, in James Elkins, On Pictures and the Words that fail them (Cambridge: Cambridge University 
Press, 1998), 3-46; Randgänge der Zeichnung, herausgegeben von Werner Busch/ Oliver Jehle/Carolin Meister (München: Fink Verlag, 2007); Räume der Zeichnung, herausgegeben von Angela Lammert und Carolin Meister (Nürnberg: Verlag für Moderne Kunst, 2007); Öffnungen. Zur Theorie und Geschichte der Zeichnung, herausgegeben von Teja Bach und Wolfram Pichler (München: Fink Verlag, 2009); On Line: Drawing through the Twentieth Century, herausgegeben von Cornelia H. Butler und Catherine De Zegher (New York: 2010).

24 Zur disegno-Theorie siehe insbesondere Wolfgang Kemp, ,,Disegno‘: Beiträge zur Geschichte des Begriffs zwischen 1547 und 1607“, Marburger Fahrbuch für Kunstwissenschaft 19 (1974): 219-240; Robert Williams, Art, Theory, and Culture in Sixteenth Century Italy. From Techne to Metatechne (Cambridge: Cambridge University Press, 1997); Carmen C. Bambach, Drawing and Painting in the Italian Renaissance Workshop: Theory and Practice, 1300 - 1600 (Cambridge: Cambridge University Press, 1999); David Rosand, Drawing Acts. Studies in Graphic Expression and Representation (Cambridge: Cambridge University Press, 2001).

$25 \mathrm{Zu}$ Leonardo immer noch grundlegend Ernst H. Gombrich, „Leonardo’s Method for Working out Composition“, in Ernst H. Gombrich, Studies in the Art of the Renaissance (London: Phaidon, 1966), 58-64. Ferner Rosand, Drawing Acts (wie in Anm. 24). Zur Tradition der „nicht-fixierenden Linie“ siehe unter anderem Werner Busch, „Die Möglichkeiten der nicht-fixierenden Linie: ein exemplarischer, historischer Abriss“, in Randgänge der Zeichnung, herausgegeben von Werner Busch/Oliver Jehle/Carolin Meister (München: Fink Verlag, 2007), 121-139; Werner Busch, Das unklassische Bild. Von Tizian bis Constable und Turner (München: C. H. Beck Verlag, 2009).

26 David Rosand, „Um 1500“, in Öffnungen. Zur Theorie und Geschichte der Zeichnung, herausgegeben von Teja Bach und Wolfram Pichler (München: Fink Verlag, 2009), 93-108.

27 Maurice Merleau-Ponty, „Le doute de Cézanne“, in Maurice Merleau-Ponty, Sens et Non-Sens (Paris: Nagel, 1948), 27. Zu Merleau-Pontys Cézanne-Interpretation vgl. Gottfried Boehm, „Der Stumme Logos“, in Leibhaftige Vernunft. Spuren von MerleauPontys Denken, herausgegeben von Alexandre Métreaux und Bernhard Waldenfels (München: Fink Verlag, 1986), 298-304; Bernhard Waldenfels, „Das Zerspringen des Seins. Ontologische Auslegungen der Erfahrung am Leitfaden der Malerei“, ebd. 144-161.

28 Gottfried Boehm, „Spur und Gespür. Zur Archäologie der Zeichnung“, in Öffnungen. Zur Theorie und Geschichte der Zeichnung, herausgegeben von Teja Bach und Wolfram Pichler (München: Fink Verlag, 2009), 43-59; Norman Bryson, 
„Ein Spaziergang um seiner selbst willen“, ebd., 27-42. Aber auch Friedrich Teja Bach und Wolfram Pichler, „Ouvertüre“, ebd., 9-26; Zur Linie siehe Sabine Mainberger, Experiment Linie. Künste und ihre Wissenschaften um 1900 (Berlin: Kadmos Verlag, 2009).

29 Hubert Damisch, Traité du Trait - Tractatus tractus (Paris: Réunion des Musées Nationaux, 1995).

30 Vgl. hierzu auch Wittgenstein PU 68 und 71.

31 Dies haben unter anderem Hans Sluga, Peter Galison und Matthias Kroß gezeigt. Peter Galison, „Aufbau/Bauhaus. Logical Positivism and Architectural Modernism,“ Critical Inquiry 16 (1990): 709-752; Hans Sluga, „Zwischen Modernismus und Postmoderne: Wittgenstein und die Architektur", in Die Wiener Fahrhundertwende, herausgegeben von Jürgen Nautz und Richard Vahrenkamp (Wien: Böhlau, 1996), 241-256; Matthias Kroß, „Die Selbstverständlichkeit der Metapher. Wittgensteins Entspannung eines sprachphilosophischen Problems“, in Wittgenstein und die Metapher, herausgegeben von Ulrich Arnswald/Jens Kertscher/Matthias Kroß (Berlin: Parerga, 2004), 23-53.

32 Kroß, „Selbstverständlichkeit der Metapher“ (wie in Anm. 31), 45.

33 Sluga, „Wittgenstein und die Architektur“ (wie in Anm. 31), 248. Ähnlich auch Kroß: „Deutet man den Tractatus als eine logische Architekturskizze und betrachtet man ihre Konstruktion als ein Modell der Wirklichkeit, dann kommt dem Philosophen die Funktion eines reinzeichnenden Baumeisters zu, der alles so lange kombiniert, bis das Modell am Ende als vollendet gelten darf.“ Kroß, „Selbstverständlichkeit der Metapher" (wie in Anm. 31), 45-46. Rüdiger Zill analysiert in seinem Aufsatz Wittgensteins Verständnis der Grenze, auch in Bezug auf Kant, für den der Begriff der Grenze eine wichtige Rolle spielt. Zill, „Der Vertrakt des Zeichners" (wie in Anm. 6).

34 Haller, „Das Kunstwerk“ (wie in Anm. 3), 31.

35 Die Veränderung von Wittgensteins Verständnis der Perspektive und des Sehens kann auf seine Beschäftigung mit der Architektur, mit dem konkreten architektonischen Körper (und auch dem menschlichen Körper im Raum) zurückgeführt werden. („Ich will wissen, was hinter mir vorgeht und drehe mich um.[...] $\underline{\text { Also }}$ ist es die Möglichkeit des mich-Umdrehens, die mir zu jener Raumvorstellung verhilft. Der Resultierende Raum um mich ist also ein gemischter Sehraum und ein Muskelgefühlsraum. Ohne das Gefühl der Fähigkeit ,mich umzudrehen' wäre meine Raumvorstellung eine wesentlich andere." (Wiener Ausgabe, Bd. I, S. 195). Vgl. hierzu Gunter Gebauer, „,Taktilität und Raumerfahrung bei Wittgenstein, “ in ARCH+. Zeitschrift für Architektur und Städtebau 157 (2001): 91-98; 
Fabian Goppelsröder, „Das Palais Stonborough. Wittgenstein als Architekt seiner Spätphilosophie“, in Wittgensteinkunst, herausgegeben von Fabian Goppelsröder (Zürich/Berlin: Diaphanes, 2006) 27-46; Nana Last, Wittgenstein's House. Language, Space and Architecture (New York: Fordham University Press, 2008).

36 Die Bedeutung der Landschaft in Wittgensteins später Philosophie wurde vor allem in der neueren Literatur betont. David Schalkwyk etwa geht in seinem äußerst inspirierenden Aufsatz auf die topographische und am Rande auch landschaftsmalerische Dimension Wittgensteins ein. Vgl. hierzu David Schalkwyk, „Wittgenstein’s ,imperfect garden“: the Ladders and Labyrinths of Philosophy as ,Dichtung““”, in The Literary Wittgenstein, herausgegeben von John Gibson und Wolfgang Huemer (London /New York: Routledge, 2004), 55-74. Trotz der medialen Differenzen gibt es einige Aspekte, die die Bereiche der (Landschafts)-Malerei/Zeichnung und Wittgensteins eigene Auffassung der Philosophie und ihrer Ziele annähern. So ist es primär die bereits in der Landschaftsmalerei der frühen Neuzeit angedeutete Einsicht der „grundsätzlichen Deutungsbedürftigkeit von Welt“, das Wissen, dass es multiple Interpretationen der Welt gibt, die durch unterschiedliche Wissenssysteme voneinander getrennt werden. Zentraler Begriff ist hier derjenige der Polyperspektivität. Im Gegensatz zur Kartographie mit ihrem Anspruch, ein wissenschaftlich objektiviertes Bild der Welt zu zeigen, ist es nicht die Intention der (Landschafts)-Malerei, absolute Objektivität zu erreichen, sondern die Mannigfaltigkeit der Erscheinungen in ihrer ganzen Komplexität zu erfassen. Diese Differenz zwischen den Ansprüchen der Kartographie und denjenigen der Landschaftsmalerei hat Tanja Michalsky in ihrem Aufsatz überzeugend dargelegt. Es sei an dieser Stelle betont, dass trotz der hier vorgenommenen Differenzierung zwischen den epistemischen Intentionen von Kartographie und Landschaftsmalerei ein Bewusstsein für die Verwischung der Grenzen sowie die gegenseitigen Befruchtungen vorhanden ist. Vgl. hierzu Tanja Michalsky, „Hic est mundi punctus et materia gloriae nostrae. Der Blick auf die Landschaft als Komplement ihrer kartographischen Eroberung“, in Das Geheimnis am Beginn der europäischen Moderne herausgegeben von Klaus Reichert (Frankfurt am Main: 2002), 436-453.

37 Man. 118, zitiert nach Pichler, Album (wie in Anm. 2).

38 PU, Vorwort, 231-232.

39 Wie Jörg Zimmermann und Manfred Frank gezeigt haben, weisen Wittgensteins Überlegungen zum Unsagbaren und seine fragmentarische Schreibweise bedeutsame Parallelen zu den poetologischen und philosophischen Schriften der Frühromantik auf. Zimmermann, Hermeneutik (wie in Anm. 3); Frank, Dichtung (wie in Anm. 3), 45. 
40 Rudolf Haller, „Wie man nicht mit dem Hammer philosophiert“, in Wittgenstein. Und. Philosophie. Literatur, herausgegeben von Wendelin Schmidt-Dengler/Martin Huber/Michael Huter (Wien: Edition S., 1990), 9-20, hier 16-17.

41 Im Anschluss an Genova, Pichler und Schalkwyk plädiere ich für eine kontextualistisch-detailorientiert und nicht systematisch-holistische Sicht des Begriffs der „übersichtlichen Darstellung“. Vgl. hierzu Judith Genova, „A Map of the Philosophical Investigations“, in Ludwig Wittgenstein. Critical Assessments, herausgegeben von Stuart Shanker (London: 1997), Bd. 2, 61; Pichler, Album (wie in Anm. 2), 190; Schalkwyk, „Imperfect garden“ (wie in Anm. 36).

42 Claus Zittel hat die Bedeutung des ästhetischen Perspektivismus für das Verständnis von Nietzsches Philosophie herausgearbeitet und dabei grundlegende Überlegungen zum ästhetischen Perspektivismus im Allgemeinen formuliert. Claus Zittel, Das ästhetische Kalkül von Friedrich Nietzsches „Also sprach Zarathustra“ (Würzburg: Königshausen und Neumann, 2000).

43 Spremberg, Aktualität (wie in Anm. 10), 189-190.

44 Bezzel, Einführung ( wie in Anm. 6), 116-117. Judith Genova hat in ihrem hervorragenden Buch ebenfalls auf diese Verbindung zwischen Zeichnen und Denken hingewiesen. Genova, A way of seeing (wie in Anm. 6), 71.

45 ,Wäre ich ein guter Zeichner, könnte ich eine unzählbare Anzahl von Ausdrücken durch vier Striche erzeugen. Wörter wie ,bombastisch' und ,getragen' könnten durch Gesichter ausgedrückt werden. Damit wären unsere Beschreibungen viel flexibler und unterschiedlicher als sie es durch den Ausdruck von Adjektiven sind.“ Ludwig Wittgenstein, Vorlesungen und Gespräche über Ästhetik, Psychoanalyse und religiösen Glauben, herausgegeben von Cyril Barret (Frankfurt am Main: Fischer Verlag, 2000), 14.

46 Die Möglichkeit, Gewohntes unter einem neuen Licht zu sehen, ist für Wittgenstein auf das Engste mit dem Aspektsehen verbunden. Das ,Aspektsehen“ als aktives Sehen führt zu einer produktiven Verarbeitung des Sichtbaren und ist für künstlerische Prozesse grundlegend. Zum Aspektsehen siehe etwa Genova, A way of seeing (wie in Anm. 6); Thorsten Jantschek, „Bemerkungen zum Begriff des Sehen-als“, in Kritik des Sehens, herausgegeben von Ralf Konersmann (Leipzig: Reclam, 1997), 299 -319. Immer noch grundlegend Joachim Schulte, Erlebnis und Ausdruck. Wittgensteins Philosophie der Psychologie (München: Philosophia Verlag, 1987).

47 Pichler hat diesen Punkt ausführlich und überzeugend dargelegt. Vgl. hierzu Pichler, Album (wie in Anm. 2).

48 Genova, „A Map of the Philosophical Investigations“(wie in Anm. 41), 62. 